\title{
Novel Gross Motor Skill Training Improves Flexibility, Reaction Time, and Mobility in Older Adults - A Pilot Study
}

\author{
Benjamin Tseng ${ }^{1,2^{*}}$, Reyanne West ${ }^{1}$, Colby Craddock ${ }^{1,2}$ and Avery Clark ${ }^{1}$ \\ ${ }^{1}$ Laboratory of Brain Aging and Neuromotor Behavior, University Blvd, Tyler, Texas, USA \\ ${ }^{2}$ Department of Health and Kinesiology, University of Texas at Tyler, Tyler, Texas, USA
}

*Corresponding author: Benjamin Tseng, Department of Health and Kinesiology, University of Texas at Tyler, Tyler, Texas, USA, Tel: +1 9035667042, +1 9035667031; E-mail: btseng@uttyler.edu

Received Date: 30 June, 2017; Accepted Date: 18 September, 2017; Published Date: 04 October, 2017

\begin{abstract}
Objective: Maintaining independence and quality of life is becoming increasingly challenging in our rapidly aging society. Emerging evidence suggests that learning novel gross motor skills that consist of multiple limb movements, hand-eye coordination, and object manipulation may be beneficial in preserving functional capacity required for daily-living activities in older adults. The purpose of this study was to investigate the impact of Bilateral Object Manipulation (BOM) training on physical function in older adults.
\end{abstract}

Methods: Thirty-six participants aged 50-70 underwent an 8-week intervention consisted of either a BOM training (BOM) of 3-ball juggling and an Exercise Training (ET). Physical function was evaluated by Range-of-Motion (ROM), Simple and Choice Reaction Times (SRT \& CRT), functional reach, and Gait Velocity $(\mathrm{GV})$. In addition, $\mathrm{GV}$ change ( $\triangle \mathrm{CV}$ ) was measured under 4 dual-task conditions where cognitive demand simultaneously competes with a motor task. Fat-Free Mass (FFM) and Fat Mass (FM) were assessed using Dual-Energy X-Ray Absorptiometry (DXA). All measures were analyzed at baseline and post-training.

Results: The BOM group showed improved upper extremity ROM $(p<0.05)$, shortened SRT and CRT $(p<0.05)$, faster GV $(p<0.05)$, and better functional reach $(\mathrm{p}<0.01)$ after training. The ET groups showed improved ROM in the lower extremity $(\mathrm{p}<0.05)$, faster $\mathrm{GV}(\mathrm{p}<0.01)$, and reduce FM $(\mathrm{p}<0.05)$.

Discussion: Our preliminary data suggest that 8 weeks of BOM training may promote better flexibility, reacting time, mobility, and dynamic balance in older adults. This finding underlines the clinical implication of a potentially cost-effective home-based therapeutic intervention in preserving daily-living function in older adults.

\section{Background and Purpose}

With advanced age comes a decline in physical function [1], which can adversely affect the ability to perform daily activities in older adults [2]. More importantly, these age-related declines can increase the risk of falls and jeopardize the independence of older adults [3]. It is projected that more than 88 million US residents are expected to be 65 or older in 2050; maintaining independence and quality of life at old age is an urgent public health concern in our rapidly aging society.

It is well established that high muscle strength helps facilitate a person's ability to maintain independence in daily-living activities, and muscle weakness has been associated with an increased incidence of falls in older people [4,5]. In addition, the effects of aerobic exercise on physical health are also well established [6-8]. However, commencing and committing to regular exercise, either aerobic or resistance training, remains a challenge to most Americans due to either physical or personal constraints. To this end, a novel home-based program that is engaging (e.g., learning a new dance routine, etc.) may provide a cost-effective alternative to conventional exercise.

To date, it is unclear if learning a new motor skill composed of a complex gross movements can promote or enhance physical functions in older adults. The purpose of this study was to 
Citation: Tseng B, West R, Clark A (2017) Novel Gross Motor Skill Training Improves Flexibility, Reaction Time, and Mobility in Older Adults - A Pilot Study. J Bone Muscles Stud 2017: 9-12. DOI : https://doi.org/10.29199/2637-7039/BOMS-101012.

investigate the effect of Bilateral Object Manipulation (BOM) training on physical function in older adults.

\section{Methods}

This study utilized a pre-test post-test design and was approved by the Human Subjects Committee of the University of Texas at Tyler. All participants provided written informed consent prior to participation in this study.

\section{Participants}

Thirty-six adults aged 50-70 from the East Texas area participated (12 male/24 female). Participants were excluded from the project if they had any of the following: (1) currently participating in any regular physical exercise or training program, (2) restricted mobility, movement disorders, or any gait or balance issues, (3) hospitalization for myocardial infarction, heart surgery, or congestive heart failure during the preceding 3 months, (4) musculoskeletal problems that would limit ability to exercise; or (5) any clinically diagnosed neurologic disorders.

\section{Outcome measures}

Measures were assessed at baseline and after 8 weeks of training:

1. Range of Motion (ROM) was assessed by measuring wrist extension, wrist flexion, shoulder flexion, hip flexion, and dorsi-flexion using a goniometer. These measurements were taken in the sited position except for hip flexion (supine). Each site was assessed 3 times; mean was calculated.

2. The Simple Reaction Time (SRT) test required participants to stop a free-falling yardstick against wall using the dominant hand before it could reach the ground when released at shoulder height at random times [9]. Distance dropped was measured in centimeters. In the Choice Reaction Time (CRT) test [9], participants were presented 2 yardsticks (1 for each hand) and were asked to stop a randomly dropped ruler without notice. Four practice trials prior to actual testing were offered to subjects in both SRT and CRT tests. Each participant was given 3 attempts; mean was calculated.

3. Gait Velocity (GV) was recorded using a 10-meter-walk test used in our previous report [10]. In addition, participants were asked to repeat the 10 -meterwalk under 4 different Dual-Task (DT) conditions designed to detect any change of gait velocity as participants walked and were presented with attentional distractions [10]. Specifically, DT-1 required subjects to generate as many words as they could that begin with letter "C" during the 10MW (letter " $C$ " verbal fluency). In DT-2, subjects were asked to remember a 5-number digit string and recite it backward during the 10MW (5-digit backward span). In DT-3, subjects were asked to perform a serial 7 subtraction task (i.e., subtracting $100-7=93 ; 93-7=86$, etc.) continuously during the $10 \mathrm{MW}$. DT-4 required subjects to recall 3 words (door, nickel, bus) that they were asked to memorize 15 minutes prior to the test and to rearrange the words in alphabetical order and say the words immediately after they were instructed to begin the 10MW. Practice rounds were given until participants reached consistent velocity at self-selected comfortable pace. During testing, one attempt was given under each DT condition. The participants' verbal responses to each DT condition were recorded, although it's not the primary focus of the present study.

4. Functional reach was tested by placing a yardstick on the wall, parallel to the floor, at the height of the acromion of the participant's dominant arm [11]. The participants were asked to stand with the feet a comfortable distance apart, and forward flex the dominant arm to approximately 90 degrees. The participants were then asked to reach forward as far as possible without taking a step or touching the wall. The distance between the start and end point was measured. Practice trials were given prior to testing to allow participants to employee the best balancing strategy without stepping. Each participant was given 3 attempts during actual testing; mean was calculated.

5. Fat-Free Mass (FFM) and Fat Mass (FM) were measured by Dual-Energy X-ray Absorptiometry (DXA) (Lunar iDXA, GE Healthcare, Madison, WI). Total FFM\% and FM\% were calculated from arms, legs, trunk, android, and gynoid regions described in previous reports [12].

\section{Intervention}

The intervention consisted of an 8-week program of a Bilateral Object Manipulation (BOM) training and an Exercise Training group (ET). The BOM group was asked to undergo the training using classic 3-ball juggling, 3 days a week for 30 minutes. The assessment of juggling ability was determined by each successful revolution of 3 balls (i.e., per cycle). Although not an outcome measure, juggling skill was evaluated at baseline and after training. The ET group was asked to exercise by walking 3 days a week for 30 minutes + light resistance training using Thera band. Weekly follow-up by phone and daily training diary were given to all participant to monitor training progress and compliance.

\section{Statistical analysis}

Statistical analysis was performed using Sigma Plot 12.5 (SSTI, San Jose, CA).

The sample size was based on our previous study of motor performance in pre Alzheimer patients which showed pronounced slowing in gait velocity under dual-task conditions by more than $20 \%$. To detect a reduction in gait velocity by 0.2 unit with standard deviation of 0.2 before and after training, we completed pre- and post- training measurements in 18 participants in each group ( $\alpha=0.05,1-\beta=0.80$, a two tailed hypothesis for ANOVA). Descriptive statistics (mean, standard deviation) were calculated and scatter plots were examined to identify outliers for each measure. Student's t-tests were conducted to detect group differences in physical fitness at baseline with significance set at $\alpha=0.05$. Lastly, two-way repeated measures Analysis of Variance 
(AVOVA) with Tukey test for pairwise comparisons was used for between and within group comparisons of changes in gait velocity under dual-task conditions

\section{Results}

Subject characteristics are presented in table 1 . No differences were found in age, gender distribution, or other physical attributes between groups at baseline.

\begin{tabular}{|c|c|c|}
\hline & BOM & ET \\
\hline Male / Female & 06-Dec & 06-Dec \\
\hline Age (years) & $66.2 \pm 5.1$ & $68.1 \pm 7.2$ \\
\hline Education (years) & $16.2 \pm 2.5$ & $16.0 \pm 3.0$ \\
\hline Height (cm) & $172.0 \pm 6.1$ & $169.3 \pm 7.0$ \\
\hline Weight (kg) & $76.4 \pm 9.0$ & $70.7 \pm 11.0$ \\
\hline Fat Mass (\%) & $34.4 \pm 6.1$ & $35.5 \pm 5.3$ \\
\hline Fat Free Mass (\%) & $30.1 \pm 9.8$ & $33.3 \pm 11.5$ \\
\hline
\end{tabular}

Table 1. Subject characteristics at baseline.

Mean $\pm \mathrm{SD}, \mathrm{BOM}=$ Bilateral Object Manipulation, ET=Exercise Training

As shown in table 2, the BOM group showed improved upper extremity ROM $(\mathrm{p}<0.05)$, shortened SRT and CRT $(\mathrm{p}<0.05)$, and faster GV $(p<0.05)$, and better functional reach $(p<0.01)$ after training; while the ET groups showed improvement ROM in the lower extremity $(p<0.05)$, faster GV $(p<0.01)$, and reduce FM $(p<0.05)$. Using two-way repeated measures ANOVA, we found significant interactions between intervention type and dual-task conditions $(\mathrm{p}<0.01, \mathrm{~F}=5.1)$. Notably, BOM participants exhibited less gait velocity declines while walking under all DT conditions compared to the ET group (Figure 1).

\begin{tabular}{|c|c|c|}
\hline & BOM & ET \\
\hline$\Delta$ Juggling $($ cycle completed) & $* 6 \pm 3$ & N/A \\
\hline$\# \Delta$ Wrist Flexion $\left(^{\circ}\right)$ & $* 6 \pm 2$ & $0 \pm 2$ \\
\hline$\# \Delta$ Wrist Extension $\left(^{\circ}\right)$ & $* 12 \pm 4$ & $0 \pm 2$ \\
\hline$\# \Delta$ Shoulder Flexion $\left({ }^{\circ}\right)$ & $* 10 \pm 2$ & $0 \pm 2$ \\
\hline$\Delta$ Hip Flexion $\left(^{\circ}\right)$ & $4 \pm 4$ & $* 8 \pm 4$ \\
\hline$\Delta$ Dorsi Flexion $\left(^{\circ}\right)$ & $2 \pm 6$ & $* 4 \pm 2$ \\
\hline$\Delta$ SRT $(\mathrm{cm})$ & $*-3.5 \pm 1.5$ & $-1.5 \pm 4.4$ \\
\hline$\Delta$ CRT $(\mathrm{cm})$ & $*-3.2 \pm 1.8$ & $-1.4 \pm 4.0$ \\
\hline$\Delta$ GV $(\%)$ & $* 8.2 \pm 3.0$ & $* * 13.0 \pm 4.0$ \\
\hline$\# \Delta$ Functional Reach $(\mathrm{cm})$ & $* * 14.0 \pm 6.0$ & $5.0 \pm 12.0$ \\
\hline$\# \Delta$ Fat Mass $(\%)$ & $0.0 \pm 6.0$ & $*-2.0 \pm 0.5$ \\
\hline$\Delta$ Fat Free Mass $(\%)$ & $1.0 \pm 9.0$ & $2.0 \pm 10.0$ \\
\hline
\end{tabular}

Table 2. Physical \& functional changes after an 8 -week intervention.

Mean $\pm \mathrm{SD}, \Delta=$ Change, BOM=Bilateral Object Manipulation, ET=Exercise Training. SRT $=$ Simple Reaction Time, $\mathrm{CRT}=$ Choice Reaction Time, $\mathrm{GV}=$ Gait Velocity

* Significant difference found compared to baseline $(\mathrm{p}<0.05)$.

**Significant difference found compared to baseline $(\mathrm{p}<0.01)$.

"Significant difference found between groups $(\mathrm{p}<0.05)$.

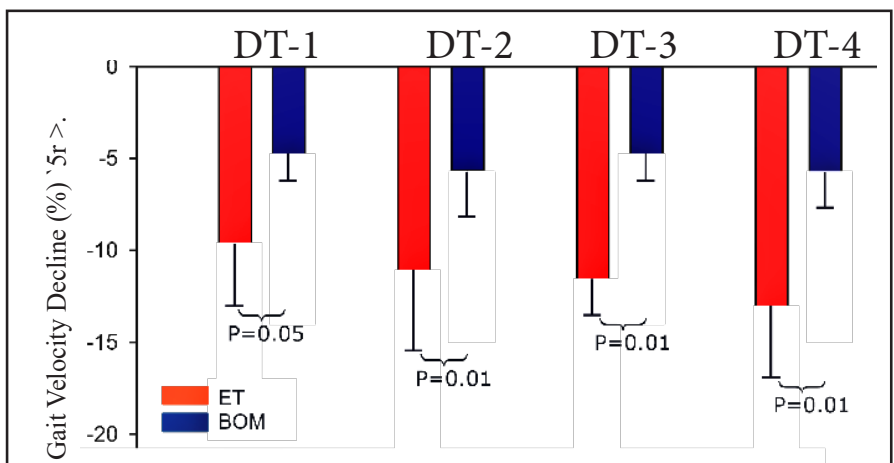

Figure 1. Walking speed under distracting conditions was better preserved in the Bilateral Object Manipulation (BOM) group compared to their Exercise Training (ET) counterparts, suggesting better resilience to attentional distractions while walking. DT1: Letter "C" Verbal Fluency, DT2: 5-Digit Backward Span, DT3: Serial 7 Subtraction, DT4: Delayed Recall \& Sorting.

\section{Discussion}

Our data suggest that 8 weeks of BOM training may be beneficial in improving range of motion, reacting time, mobility, and dynamic balance in older adults.

Specifically, the BOM group showed improved flexibility in the upper extremities.

Namely, we found increased ROM in wrist flexion, wrist extension, and shoulder flexion. To date, information regarding juggling maintaining or increasing range of motion in the arms and shoulder remain scarce although many claims persist. It is our speculation that juggling may promote better ROM it utilizes body mechanics otherwise rarely engaged. In addition, joint flexibility shares the same "use it or lose it" principle as commonly practiced in maintaining muscle mass and cardiovascular fitness.

To our Best knowledge, this is the first trial to report improvement in joint range of motion in wrist and shoulder after a BOM training by juggling. As hypothesized, the ET group showed increased ROM in hip flexion and dorsiflexion after 8 weeks of walking and Thera band exercise training. This result is consistent with previous studies that investigated the effect of exercise on joint range of motion in the lower extremities [13]. In addition, we found no difference in FFM and FM in the BOM group; while the ET group showed reduced fat mass by $2 \%(\mathrm{p}<0.05)$.

The BOM group showed improved reaction time in both simple- and choice reaction time tests. Of note, the classic 3-ball cascade juggling has been previously reported to promote brain grey matter growth [14]. Although the underlying mechanism involved is unclear, it is our speculation that there may be a relationship between the improved reaction times and enhanced neuroplasticity as the result of BOM training.

Although both groups showed improved gait velocity after training, we found that the BOM group out-performed the ET group while walking under distracting conditions (Figure 1). A previous study that conducted 8-week combined high-intensity aerobic and strength training vs. gross motor activities found 
Citation: Tseng B, West R, Clark A (2017) Novel Gross Motor Skill Training Improves Flexibility, Reaction Time, and Mobility in Older Adults - A Pilot Study. J Bone Muscles Stud 2017: 9-12.

that different exercise programs targeting physical fitness and/or gross motor skills may lead to equivalent improvement cognitive functions in a cohort of healthy older adults [15]. In the present study, we found that gait velocity under distracting conditions was better preserved in participants who underwent the BOM training, suggesting a better resilience to attentional distraction while walking during daily activities in real life. In addition, the BOM group showed superior dynamic balance as shown by the functional reach test. These findings may yield clinical significance because slow walking speed, gait variability, and poor dynamic balance are the biggest identified fall risks [16].

It was not surprising that no FM\% \& FFM\% change was found in the BOM group. Although the BOM training requires effort and attention, it was not particularly physically demanding. The nature of BOM training may potentially attribute to this finding due to negligible caloric expenditure. In contrast, the ET group show global fat mass loss after 8 weeks. Given that all participants were aerobically sedentary prior to this study, it is likely that our walking and resistance program increased participants' total energy expenditure, which may promote weight and/or fat loss assuming that there was no significant dietary change.

We acknowledge the limitation of a small sample size of this interventional study and note that the results should be interpreted with caution. We implemented a stringent screening protocol to control for potential confounding factors such as comorbidity and aerobic fitness level which may influence fitness level in older adults. In addition, sex, age, educational and body mass index between groups were similar. We also acknowledge the lack of control group in this study. Inferences made in this study were based on comparisons between the BOM and exercise training groups. It was our assumption that no changes were expected in a control group without any training.

Of note, although participants' juggling skill improved after BOM training, it is imperative to emphasize that the primary focus of this study was to demonstrate measureable transfer functions in physical aspects that are useful in daily activity setting (e.g., flexibility, reaction time, and balance) as the result of bilateral object manipulation training via a central mechanism.

Promoting physical health and maintaining the functional capacity of daily activities is imperative in our aging society. As demonstrated in this study, 3-ball juggling is a complex skill that can challenge participants in several physical aspects to meet a higher demand. Our data suggest that 8 weeks of bilateral object manipulation training may be beneficial in improving flexibility, reacting time, mobility, and dynamic balance in older adults. Future investigation should aim to validate the physiologic mechanisms involved in the training adaptations observed in the current study.

To our knowledge, this was the first investigation to explore the feasibility of a home-based training alternative to the conventional exercise training in preserving overall physical function in older adults. This finding underlines the clinical implication of a potentially cost-effective therapeutic intervention in preserving daily-living function in older adults.

\section{References}

1. Seidler RD, Alberts JL, Stelmach GE (2002) Changes in multi-joint performance with age. Motor control 6: 19-31.

2. Seidler RD, Bernard JA, Burutolu TB, Fling BW, Gordon MT, et al. (2010) Motor control and aging: Links to age-related brain structural, functional, and biochemical effects. Neurosci Biobehav Rev 34: 721-733.

3. Maki BE, Mcllroy WE (1996) Postural control in the older adult. Clin Geriatr Med 12: 635-658.

4. Hunter GR, McCarthy JP, Bamman MM (2004) Effects of resistance training on older adults. Sports Med 34: 329-348.

5. Moxley Scarborough D, Krebs DE, Harris BA (1999) Quadriceps muscle strength and dynamic stability in elderly persons. Gait Posture 10: $10-20$.

6. Tanaka H, Seals DR (2003) Invited Review: Dynamic exercise performance in Masters athletes: insight into the effects of primary human aging on physiological functional capacity. J Appl Physiol 95: 2152-2162.

7. Taguchi N, Higaki Y, Inoue S, Kimura H, Tanaka K (2010) Effects of a 12-month multicomponent exercise program on physical performance, daily physical activity, and quality of life in very elderly people with minor disabilities: an intervention study. J Epidemiol 20: 21-29.

8. Bhella PS, Hastings JL, Fujimoto N, Shibata S, Carrick-Ranson G, et al. (2014) Impact of lifelong exercise "dose" on left ventricular compliance and distensibility. J Am Coll Cardiol 64: 1257-1266.

9. Mercer VS, Hankins CC, Spinks AJ, Tedder DD (2009) Reliability and validity of a clinical test of reaction time in older adults. J Geriatr Phys Ther 32: 103-110.

10. Tseng BY, Cullum CM, Zhang R (2014) Older adults with amnestic mild cognitive impairment exhibit exacerbated gait slowing under dual-task challenges. Curr Alzheimer Res 11: 494-500.

11. Duncan PW, Weiner DK, Chandler J, Studenski S (1990) Functional reach: a new clinical measure of balance. J Gerontol 45: 192-197.

12. Kang SM, Yoon JW, Ahn HY, Kim SY, Lee KH, et al. (2011) Android fat depot is more closely associated with metabolic syndrome than abdominal visceral fat in elderly people. PLoS One 6: 27694.

13. van der Wees P, Lenssen AF, Hendriks E, Stomp E, Dekker J (2006) Effectiveness of exercise therapy and manual mobilisation in ankle sprain and functional instability: a systematic review. Aust J Physiother 52: 27-37.

14. Draganski B, Gaser C, Busch V, Schuierer G, Bogdahn U, et al. (2004) Neuroplasticity: changes in grey matter induced by training. Nature 427: 311-312

15. Berryman N, Bherer L, Nadeau S, Lauzière S, Lehr L, et al. (2014) Multiple roads lead to Rome: combined high-intensity aerobic and strength training vs. gross motor activities leads to equivalent improvement in executive functions in a cohort of healthy older adults. Age (Dordr) 36: 9710.

16. Roos PE, Dingwell JB (2013) Using Dynamic Walking Models to Identify Factors that Contribute to Increased Risk of Falling in Older Adults. Human Movement Science 32: 984-996. 